\title{
Patient and clinician satisfaction with video consultations in dentistry - part two: clinician satisfaction
}

\author{
Kate Parker*1 and Matthew Chia ${ }^{1}$
}

\section{Key points}

Assesses clinician satisfaction with video clinics in orthodontics.
Highlights how video clinics were used during the COVID-19 pandemic.
Identifies areas where clinicians felt using video clinics were most appropriate for use within orthodontics.

\begin{abstract}
Introduction Video consultations are currently not commonly used in dentistry; therefore, most clinicians lack experience in using this technology and seeing patients in this way. When implementing video clinics, it is important to assess clinician satisfaction as well as patient satisfaction to allow for developments and improvements, and to ensure that the highest levels of care are provided.
\end{abstract}

Aim To assess patient and clinician satisfaction with the Attend Anywhere video consultations used by the orthodontic team at Croydon University Hospital.

Method Two separate satisfaction questionnaires were piloted and developed: one for patients and one for clinicians. For all patients scheduled for a video consultation between 15 May 2020 and 15 June 2020 in the orthodontic department, all clinicians were invited to complete a clinician satisfaction questionnaire at the end of the consultation to assess satisfaction with the encounter.

Results In total, 114 patients attended video consultations, for which there were 128 clinician completed questionnaires, as some patients were seen by multiple clinicians on multidisciplinary clinics. Over $89 \%$ of clinicians strongly agreed or agreed with all of the satisfaction statements, with the highest levels of satisfaction being for ease of use and convenience. Over $95 \%$ of clinicians strongly agreed or agreed that they would recommend using video consultations and over $89 \%$ preferred using a video consultation, if appropriate, to a face-to-face appointment. Overall, the highest levels of satisfaction were for retainer reviews.

Conclusion High levels of clinician satisfaction were found with the telemedicine clinics in orthodontics across a range of different appointment types and encounters. Satisfaction varied according to the type of appointment; therefore, this should be a factor for consideration when planning the most appropriate use of telemedicine clinics going forwards.

\section{Introduction}

Part one of this article discussed the use of telemedicine with its associated advantages and limitations, and presented the results for patient satisfaction with the telemedicine clinics carried out by the orthodontic team at Croydon University Hospital. Part two of this article focuses on clinician satisfaction with the same telemedicine clinic interactions involving the patients from the first part of the study.

The use of telemedicine is relatively new in dentistry, so establishing telemedicine clinics in

${ }^{1}$ Croydon University Hospital, 530 London Road, Thornton Heath, CR7 7YE, UK.

${ }^{*}$ Correspondence to: Kate Parker

Email address: kate.parker3@nhs.net

Refereed Paper.

Accepted 9 September 2020

https://doi.org/10.1038/s41415-021-3009-9 orthodontics requires clinicians to be flexible, develop new skills and adapt to a new way of working. ${ }^{1}$ As many clinicians have little or no experience of using telemedicine, they may be apprehensive about using this new technology and about the move away from traditional face-to-face appointments. There is evidence that patients find web-based communication acceptable and welcome a move towards an increased use of this style of consultation. ${ }^{2,3,4,5,6,7}$

As discussed in part one of the article, there are numerous benefits to telemedicine, but a specific advantage for clinicians is the ability to assess visual information and gain diagnostic signs from the patient which would not be possible with a telephone call. ${ }^{8}$ There are also acknowledged limitations and barriers to the use of telemedicine, including technological problems, setup costs, staff training and decreased interaction of patients with healthcare professionals. ${ }^{9}$
Video consultations with patients are only beneficial when patients are unable to or do not need to attend a face-to-face appointment, if the same standards of care can be achieved remotely. ${ }^{10,11}$ Telemedicine is therefore only appropriate in certain circumstances and this is emphasised by the General Medical Council statement that 'digital and technological advances may present a more convenient way for patients to access healthcare, but it is important that these services do not compromise standards of care and patient safety.' ${ }^{12}$ This is a valid concern for clinicians. ${ }^{2,3,4,5,6,7}$

When implementing new systems and different practices of working, both patient and clinician feedback is necessary to ensure that the services provide the best possible patient care and high levels of satisfaction for patients and clinicians alike. ${ }^{13}$ It also provides a starting point for developing and improving the service. 
Aims

This service evaluation aimed to assess patient and clinician satisfaction with the Attend Anywhere telemedicine clinics used by the orthodontic team at Croydon University Hospital. The focus of this paper is to examine clinician satisfaction.

\section{Method}

Two questionnaires were used to assess satisfaction: one for patients and the other for clinicians. Both questionnaires were specifically developed for this study, piloted and modified before use. Each one consisted of nine questions, with the majority focusing on the same topic areas to assess patient and clinician satisfaction with the same telemedicine clinic interaction.

For the clinician questionnaire, the first question asked the clinician details of the type of appointment they were using the video consultation for. The next eight questions aimed to assess the clinician's experience of the video consultation and asked the clinician to rate eight statements using a five-point Likert scale (strongly agree, agree, neither agree nor disagree, disagree, strongly disagree)..$^{14}$ Clinicians were asked to rate the following statements:

- The video consultation was easy to use

- The video consultation was convenient

- I was able to assess my patient

- I was able to discuss my patient's care with them

- I was able to maintain an acceptable standard of care for my patient

- Using the video consultation has been a positive experience

- I would recommend using video consultations to other clinicians

- Where clinically appropriate, I would prefer to use video consultations instead of a faceto-face appointment.

Following this, there was a free-text section for comments and suggestions.

As detailed in part one of this article, 128 consecutive patients scheduled for an appointment with the orthodontic team between 15 May 2020 and 15 June 2020 were contacted by telephone to offer them a video consultation to replace their planned faceto-face appointment, due to the COVID-19 pandemic. Of these patients, eight declined a video consultation and were scheduled instead for a telephone consultation. A further six patients failed to 'attend' their video consultation. This resulted in 114 patients being seen for a video consultation between 15 May 2020 and 15 June 2020. The video consultations were carried out with the clinician who was providing the patient's care, the patient and a parent if the patient was under the age of 18 years old or if the patient wanted a parent to be present. Verbal consent was obtained and recorded in the clinical notes. For multidisciplinary clinic (MDT) video consultations with more than one professional, to ensure that the opinions of all participants were captured, each attending clinician completed a separate questionnaire for the same patient encounter.

At the end of the video consultation, the survey automatically displayed on each clinician's screen for them to complete. Clinicians were reassured that their participation in the questionnaire was voluntary. It was explained to clinicians that by completing the questionnaire, they were consenting to participate in the service evaluation. After each clinic, the administrative team sent a reminder e-mail to all clinicians, inviting them to complete the questionnaire for their video consultations, if they had not already done so.

The questionnaire was hosted by Survey Monkey, which collated the data anonymously. Data were then analysed using Survey Monkey and Microsoft Excel.

\section{Results}

Between 15 May 2020 and 15 June 2020, 114 patients had video consultations, for which 128 clinician satisfaction questionnaires were completed (100\% clinician response rate). The 'failure to attend' rate for video consultations was $5.3 \%$ (six patients).

Six clinicians took part in the surveys. The clinicians involved represented a wide range of experience from orthodontics and oral surgery (for the MDT consultations), including general dental practitioner, staff grade, senior speciality registrar, associate specialist and consultant. The video consultations were carried out for a range of reasons, including: fixed appliance review (43.8\%); MDT consultation (22.7\%); retainer review (17.2\%); removable or functional appliance review (5.5\%); review of dental development (4.6\%); and a small number of patients were seen for new patient consultations (1.6\%). Additionally, $4.6 \%$ of patients had video consultations for 'other' reasons, which included giving oral hygiene instruction or emergency advice.

The results for clinician satisfaction with the video consultations are shown in Figures $1,2,3,4,5,6,7$ and 8 . Figure 1 shows that the majority of clinicians felt that the video consultation was easy to use, with the majority of clinicians either strongly agreeing (79.7\%) or agreeing $(14.1 \%)$ with this statement.

The majority of clinicians also either strongly agreed $(84.4 \%)$ or agreed $(12.5 \%)$ that the video consultation was convenient, with only a small number neither agreeing nor disagreeing (2.3\%) or disagreeing (0.8\%) (Fig. 2).

As shown in Figure 3, the results were more dispersed for whether clinicians felt they were able to assess their patient using the video consultation. Here, $53.1 \%$ of clinicians strongly agreed and $37.5 \%$ agreed. Although no clinicians strongly disagreed, $4.7 \%$ disagreed and $4.7 \%$ neither agreed nor disagreed.

The majority of clinicians strongly agreed that via the video consultation, they were able to discuss their patient's care with them (78.9\%), with another $18.0 \%$ agreeing with the statement. No clinicians neither agreed nor disagreed; however, $3.1 \%$ did disagree with this statement (Fig. 4).

Figure 5 shows the results for whether clinicians felt they were able to maintain an acceptable standard of care for their patient through the video consultation. The majority of clinicians either strongly agreed (57.0\%) or agreed (36.7\%) with this statement. Only a small number of clinicians neither agreed nor disagreed or disagreed, and none strongly disagreed.

The majority of clinicians reported that using the video consultation had been a positive experience, with very few clinicians disagreeing $(1.6 \%)$ or neither agreeing nor disagreeing (4.7\%) (Fig. 6).

Similar results were found for whether clinicians would recommend the use of video consultations to other clinicians (Fig. 7). Here, $73.2 \%$ strongly agreed and $22.1 \%$ agreed.

Figure 8 shows the results for whether clinicians, where appropriate, would prefer to use video consultations instead of a face-toface consultation. It can be seen that the results are more spread out for this statement, with less clinicians strongly agreeing $(60.2 \%)$ and agreeing $(29.7 \%)$ than for the other aspects of clinician satisfaction. More clinicians also felt that they neither agreed nor disagreed (8.6\%) than for the other areas assessed by the questionnaire. 


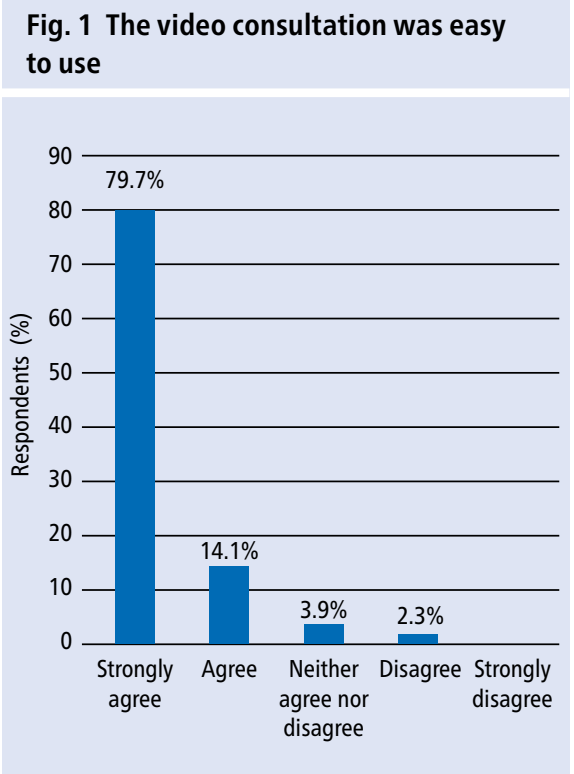

\section{Fig. 4 I was able to discuss my patient's} care with them

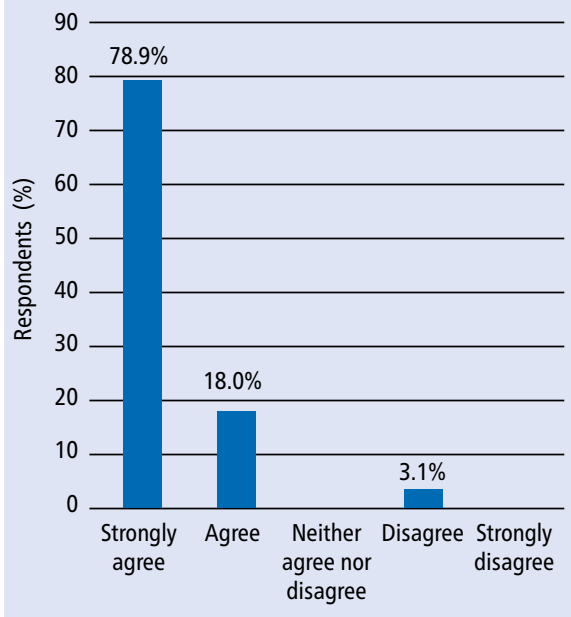

Clinician satisfaction was further assessed according to the three main reasons for patient attendance: retainer reviews, arch wire reviews and MDT consultations. Figure 9 shows that more clinicians would strongly agree to recommend using a video consultation for retainer reviews (90.8\%) than for arch wire reviews $(80.3 \%)$ or MDT consultations (44.8\%).

The results for whether clinicians would prefer to use a video consultation instead of a face-to-face appointment, if clinically appropriate, were also assessed according to the patient's reason for attendance (Fig. 10). Here, it can be seen that, again, more clinicians strongly agreed with this statement for retainer reviews $(81.8 \%)$ than for arch wire reviews (62.5\%) or MDT consultations (34.5\%).
Fig. 2 The video consultation was convenient

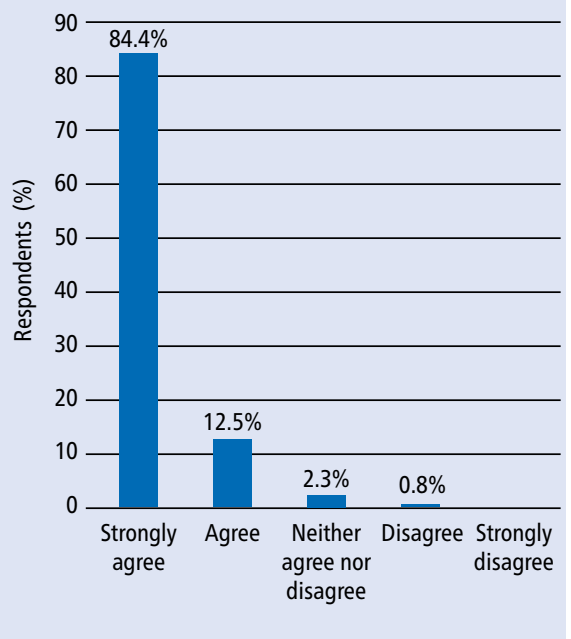

Fig. 5 I was able to maintain an acceptable standard of care for my patient

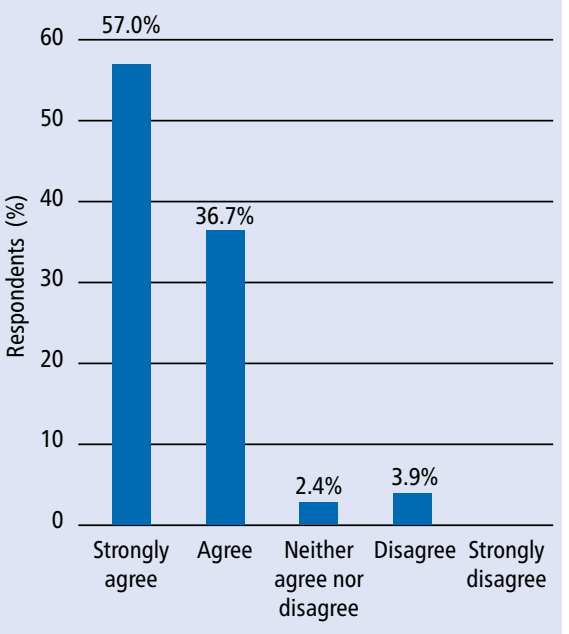

Fig. 7 I would recommend using video consultations to other clinicians

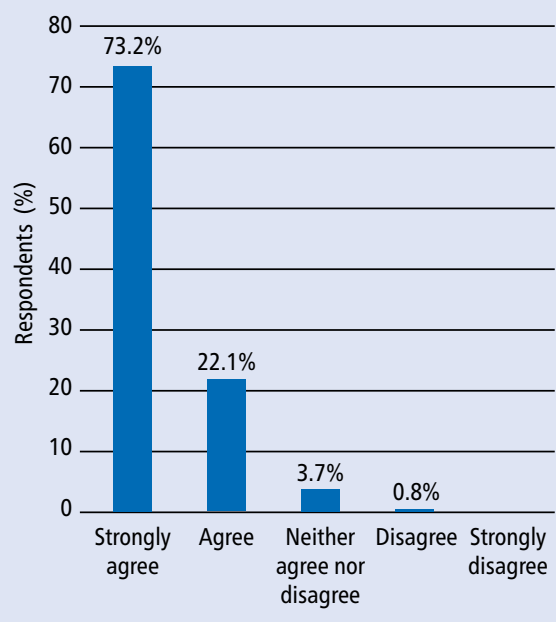

Fig. 3 I was able to assess my patient

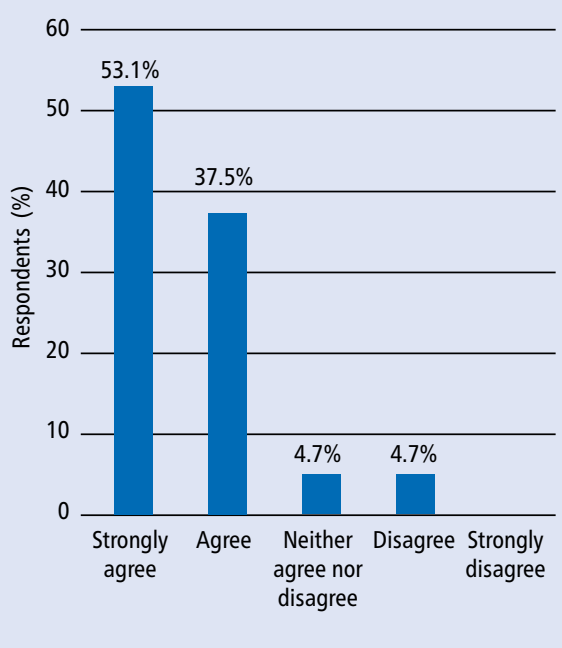

Fig. 6 Using the video consultation has been a positive experience

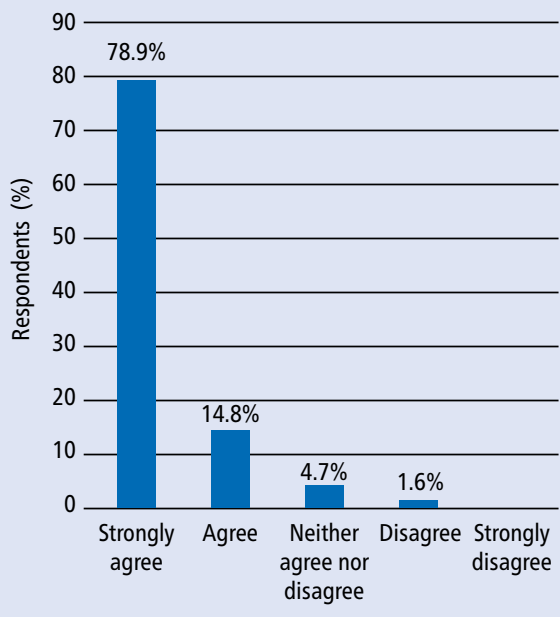

Fig. 8 Where clinically appropriate, I would prefer to use video consultations instead of a face-to-face appointment

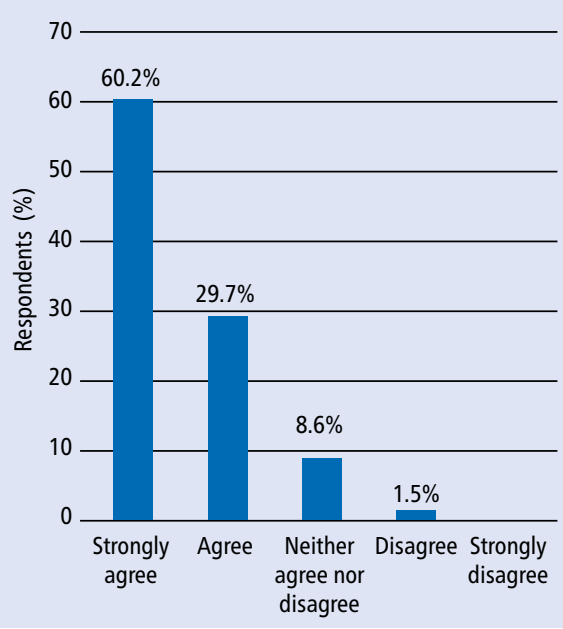




\section{Fig. 9 I would recommend using video consultations to other clinicians (results according to reason for attendance)}

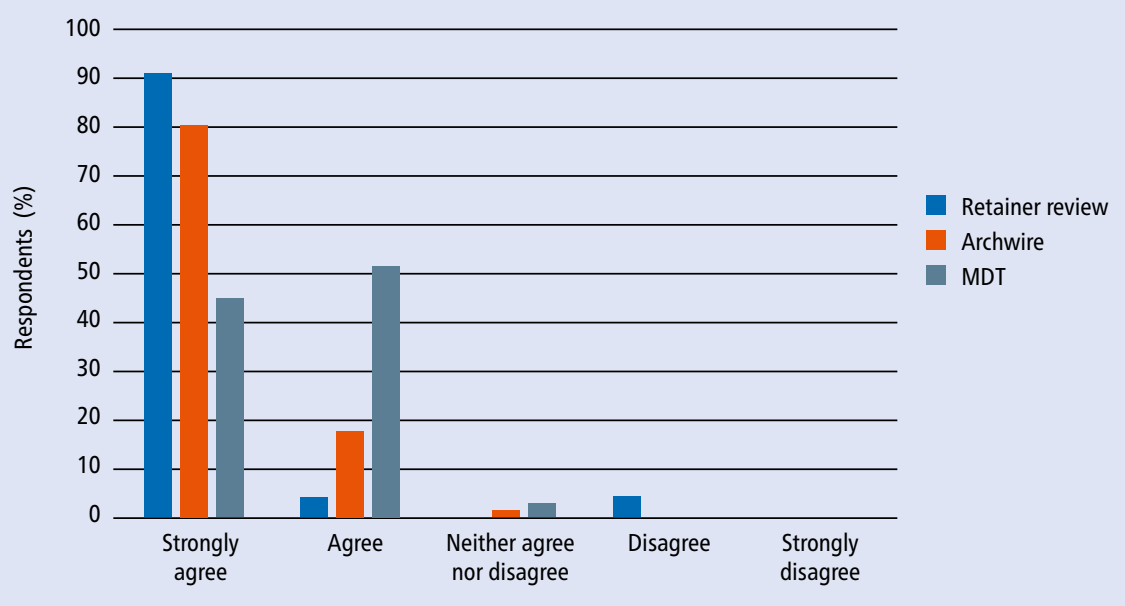

Fig. 10 Where clinically appropriate, I would prefer to use video consultations instead of a face-to-face appointment (results according to reason for attendance)

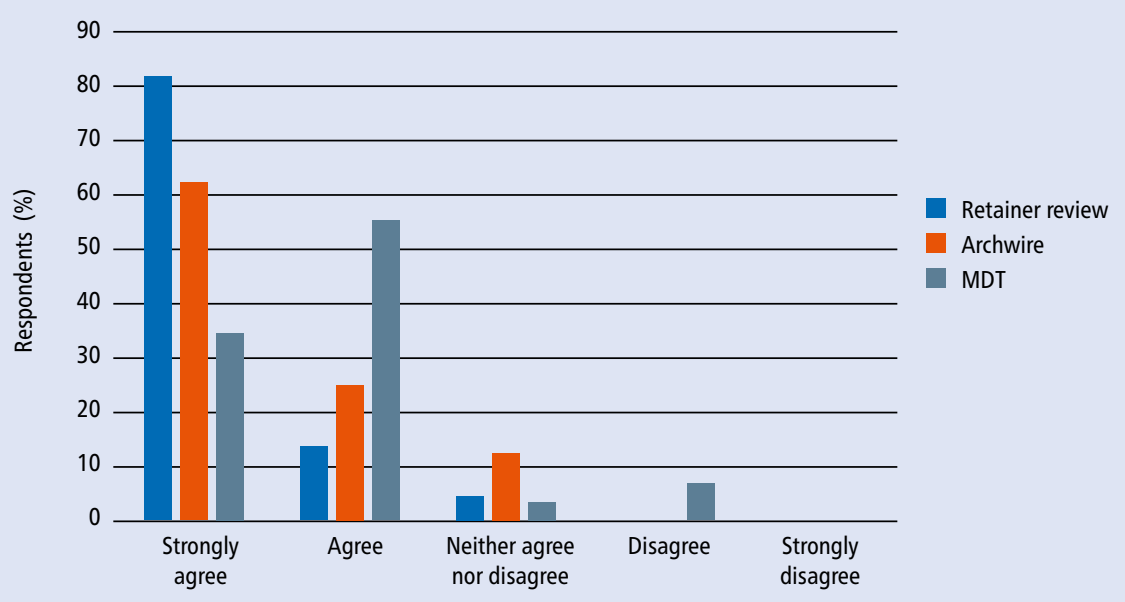

Box 1 shows a selection of the clinicians' comments regarding the video consultations. It can be seen that there is a range of observations, which highlights the positive aspects of the video consultations, including clear connection, ease of use, good assessment of dental development and ability to perform simple procedures, such as retainer and removable appliance reviews. The comments also recognise the limitations of telemedicine, such as the reliance on appropriate technology and not always being able to fully assess the patient's occlusion, especially posteriorly if the lighting or image quality is poor.

\section{Discussion}

This study has shown there to be generally high levels of clinician satisfaction with the use of telemedicine for orthodontic patients across a range of different appointment types and encounters. The results also applied across a spectrum of different clinicians from two different specialties. For all of the satisfaction statements, over $89 \%$ of clinicians either strongly agreed or agreed, no clinicians strongly disagreed, and less than 5\% disagreed with any of the statements.

The areas of whether the video consultations were easy to use and convenient scored highly and confirm the previously reported advantages from the medical profession, such as improved efficiency and quality of service. ${ }^{2,3,6,7}$ The capacity to assess patients and discuss their care with them had high levels of agreement. This reinforces the improved diagnosis and enhanced communication that comes with the addition of the visual element when providing care using this modality compared to a purely auditory telephone call. ${ }^{8}$ These key elements all
Box 1 A selection of the clinician comments for their video consultations

- Very clear. Could easily assess patient for the need to expose and bond of a canine which remained unerupted

- Very clear connection and could assess patient well to plan for a frenectomy

- Good assessment for post-op review

- Difficult to hear second clinician on the video call

- Able to discuss radiographic findings

- Able to assess canine development

- Good assessment of retainer and post-op alignment

- Sound and video connection was poor at the patient end and unable to fully assess. However, could use the chat function

- Able to prescribe class II elastics

- Lighting from the patient's perspective could have been better. I think they were using a mobile as opposed to a computer. Sound was good. Able to speak to both patient and mum

- Able to assess supernumerary and requirement for $\mathrm{CBCT}$ scan

- Able to assess anterior teeth but not able to properly assess patient's occlusion. Ideal for discussion [sic] treatment pathway/future treatment, answering queries

- Good retainer check

- Able to assess functional appliance progress especially overjet and overbite reduction

- Able to update patient on CBCT findings

- Good assessment of elastic use

- Very clear audio and visual connection

- Quality of call is dependent on correct technology from the patient end

- Shared BOS [British Orthodontic Society] retainer video with the patient

- The quality of the picture from the patient was limited and not always clear.

lead to the ability to maintain high standards of care, as demonstrated by good agreement with the satisfaction statement in this area. This may start to positively address the concerns that clinicians may have with regards to telemedicine compromising standards of care.

Although there were high levels of satisfaction reported for 'if clinicians felt able to assess their patients', in retrospect, it may have been more appropriate for this question to have required a 'yes/no' answer rather than being answered using the five-point Likert scale. This would 
have allowed a more definitive assessment of whether clinicians felt able to assess their patients appropriately. This would enable a more robust evaluation of when it may be appropriate to use a video consultation instead of a face-to-face appointment. Further work to develop this would include a 'verification' faceto-face consultation to assess the accuracy of the video consultation, as reported by Martin et al. ${ }^{15}$ This would provide more confidence and clarity for clinicians to ensure that their clinical responsibilities and duty of care may be fulfilled.

The strong agreement seen with the statements around positive experience, recommending the use of video consultations and having a preference for video consultations provides more evidence for the overall advantages for clinicians. This shows that it is a rewarding and beneficial form of communication for the individual, which should encourage the professional to develop and learn further skills in this area.

Clinician satisfaction scores varied according to the encounter type, with the highest levels of satisfaction reported for retainer reviews, then arch wire reviews, followed by MDT consultations. This is also reflected in the comments, with individuals recognising the limitations of telemedicine where active treatment is required. As retainer reviews often require little or no mechanical treatment, it is probable that this may contribute to clinicians' confidence in carrying these out with video consultations. The nature of the treatment required for arch wire changes means that it is unlikely that video consultations can be used for long periods of time. However, with careful consideration and innovation, video consultations may be incorporated into active orthodontic treatment at defined and predictable stages, such as prescription and supervision of intraoral elastics, monitoring compliance with appliances or overseeing space closure. However, it is incumbent on clinicians, with peer review, to have insight on the restrictions of video consultations and to define the parameters of care which they can provide while maintaining high standards of care.

Although no active treatment is provided at MDT consultations, due to multiple clinicians being on the video consultation, this may reduce the quality of the audio and visual feedback of these calls and thus be reflected in the decreased clinician satisfaction. Another consideration for our MDT consultations was a thorough, detailed preparation protocol (including records and radiographs, as necessary) of specific cases that have clear management strategies. Our guidance also required the patient to have previously been examined in person by a senior member of the team before requiring the video consultation. These cases could then be assessed easily using a video consultation; for example, assessment for frenectomies, surgical exposures or monitoring dental development. However, patients that require complex planning would still require a face-to-face consultation and may not be suitable for a video consultation.

When considering the use of telemedicine in the wider areas of dentistry, it is important to appreciate the benefits and limitations of telemedicine with the aim of delivering safe and appropriate care. Therefore, it is advisable that patients are pre-screened to assess their suitability for a video consultation. This will aim to ensure that the interaction is effective, efficient and beneficial to both the patient and the clinician. Selecting only appropriate cases will also help to maintain high levels of satisfaction for everyone involved in the consultation and ensure that high standards of care are maintained.

The low number of patients that 'did not attend' their video consultation highlights another advantage of telemedicine for clinicians - reducing non-attendance. The 5.3\% 'failure to attend' rate compares favourably to $23 \%$ non-attendance rates across medical specialties for face-to-face appointments. ${ }^{16}$

This study focused on clinician satisfaction with video consultations in orthodontic care and, therefore, the results can be used to draw firm conclusions regarding orthodontics. However, these experiences could be considered applicable in other areas of dentistry as there are elements that are not exclusive to orthodontics. This data can provide a solid groundwork for future research in this area in the profession to help develop and advance the future applications of telemedicine in dentistry.

\section{Conclusion}

This study has shown there to be generally high levels of clinician satisfaction with the use of telemedicine clinics in orthodontics across a range of different appointment types and encounters. Satisfaction varied according to the appointment type; therefore, this should be a factor for consideration when planning the most appropriate use of telemedicine clinics going forwards. However, the results have proved that video consultations can be considered in the modern armamentarium of technology to improve patient care and enhance communication in everyday clinical practice.

\section{Conflict of interest}

The authors declare no conflicts of interest.

\section{Acknowledgements}

The authors would like to thank: Julia Radecki, Renu George, Sara Stephens and Varlie Bacon for their assistance with this project.

\section{References}

1. Kreps $G$ L, Neuhauser L. New directions in eHealth communication: Opportunities and challenges. Patient Educ Couns 2010; 78: 329-336.

2. Haluza D, Jungwirth D. ICT and the future of health care: aspects of health promotion. Int J Med Inform 2015; 84: 48-57.

3. Peeters J M, Krijgsman J W, Brabers A E, Jong J D, Friele R D. Use and uptake of eHealth in general practice: a cross-sectional survey and focus group study among health care users and general practitioners. JMIR Med Inform 2016; D0I: 10.2196/ medinform. 4515 .

4. Ariens L F, Schussler-Raymakers F M, Frima C et al. Barriers and facilitators to eHealth use in daily practice: perspectives of patients and professionals in Dermatology. J Med Internet Res 2017; DOI: 10.2196/ jmir.7512.

5. Ruiz Morilla M D, Sans M, Casasa A, Giménez N. Implementing technology in healthcare: Insight from physicians. BMC Med Inform Decis Mak 2017; 17: 92.

6. Lupiáñez-Villanueva F, Hardey M, Torrent J, Ficapal P. The integration of Information and Communication Technology into medical practice. Int J Med Inform 2010; 79: 478-491.

7. Illiger K, Hupka M, von Jan U, Wichelhaus D, Albrecht U. Mobile technologies: Expectancy, usage, and acceptance of clinical staff and patients at a university medical centre. JMIR Mhealth Uhealth 2014; DOI: 10.2196/mhealth.3799.

8. Greenhalgh T, Koh G C H, Car J. Covid-19: A Remote Assessment in Primary Care. BMJ 2020; DOI: 10.1136/ bmj.m1182.

9. Parker K, Chia M. Remote working in dentistry in a time of crisis - tools and their uses. Dent Update 2020; 47: 515-526.

10. Gray LC, Edirippulige S, Smith A C et al. Telehealth for nursing homes: the utilization of specialist services for residential care. J Telemed Telecare 2012; 18: 142-146.

11. Chan W M, Woo J, Hui E, Hjelm N M. The role of telenursing in the provision of geriatric outreach services to residential homes in Hong Kong. J Telemed Telecare 2001; 7: 38-46

12. General Medical Council. Regulatory Approaches to Telemedicine. 2018. Available at https://www.gmc-uk. org/about/what-we-do-and-why/data-and-research/ research-and-insight-archive/regulatory-approachesto-telemedicine (accessed May 2020).

13. Al-Abri R, Al-Balushi A. Patient satisfaction survey as a tool towards quality improvement. Oman Med J 2014; 29: 3-7.

14. Likert R. A technique for the measurement of attitudes. Arch Psychol 1932; 22: 1-55.

15. Martin N, Shahrbaf S, Towers A, Stokes C, Storey C. Remote clinical consultations in restorative dentistry: a clinical service evaluation study. Br Dent J 2020; 228: 441-447.

16. Dantas L F, Fleck J L, Oliveira F L, Hamacher S No-shows in appointment scheduling - A systematic literature review. Health Policy 2018; 122: 412-421. 